\title{
Space-Time Processing with Channel Knowledge at the Transmitter
}

\author{
Joon Hyun Sung and John R. Barry \\ School of Electrical and Computer Engineering \\ Georgia Institute of Technology \\ Atlanta, Georgia 30332-0250, USA \\ \{jhsung, barry\}@ee.gatech.edu
}

\begin{abstract}
We investigate how knowledge of the channel at the transmitter can improve performance in array-to-array wireless communications. We explore the practical issues associated with space-time processing based on a singular-value decomposition (SVD) of the channel matrix. Compared to space-time block codes that do not require channel knowledge at the transmitter, the proposed method without channel coding requires $8.3 \mathrm{~dB}$ less SNR per antenna to achieve $6 \mathrm{bits} / \mathrm{sec} / \mathrm{Hz}$ with 4 transmit and 4 receive antennas. Exploiting the properties of the SVD, we propose fixed bit and power allocations that avoid dynamic allocations. We also describe how reciprocity in a timedivision duplex system may be exploited to simplify implementation of the SVD processing.
\end{abstract}

\section{Introduction}

The introduction of antenna arrays at the transmitter and receiver can dramatically improve the capacity of a wireless communication system [1]. When the transmitter does not have channel knowledge, many transmit diversity techniques have been reported, including the layered space-time scheme of Foschini [2] and the space-time trellis codes of Tarokh et al. [3]. Alamouti proposed a simple and effective block code for transmission using two transmit antennas [4], which was generalized to orthogonal space-time block codes (STBC) by Tarokh et al. [5][6].

In some applications, the transmitter knows the channel because of either explicit feedback or channel symmetry in time-division duplex (TDD) systems. In such cases, the transmitter can exploit this knowledge to achieve a higher transmission rate. It has been known for decades that the capacity is achieved when the channel is diagonalized by the singular-value decomposition (SVD) and the power is distributed by the water-pouring procedure [7][8].

In this paper, we investigate the performance of the SVD-based scheme in terms of bit-error rate (BER) using quadrature amplitude modulation (QAM) constellations and a practical bit and power allocation algorithm, and compare it to the performance of STBC. Instead of distributing power and bits adaptively according to the channel gains, we explore the possibility of a fixed allocation, and show that the resulting performance penalty is small. We also

This work was supported in part by the Yamacraw mission

(http://www.yamacraw.org) and by NSF grant no. CCR-0082329. describe how reciprocity in TDD systems simplifies implementation.

\section{System Model}

We consider the array-to-array system shown in Fig. 1-a, consisting of a transmitter with $N$ antennas and a receiver with $M$ antennas, and denoted as an $M \times N$ system. Let $x_{k}^{(j)}$ denote the complex QAM symbol transmitted by the $j$-th antenna during the $k$-th signaling interval of duration $T$. The $N$ symbol sequences $x_{k}^{(1)}$ through $x_{k}^{(N)}$ drive identical pulse-shape filters $g(t)$, assumed here to have zero excess bandwidth. The signals are then upconverted to a carrier frequency $f_{0}$ and transmitted across a quasi-static fading channel, so that the signal emitted by the $j$-th antenna is $\operatorname{Re}\left\{e^{j 2 \pi f_{0} t} \sum_{k} x_{k}{ }^{(i)} g(t-k T)\right\}$. At the receiver, the $M$ passband observations are downconverted, filtered by $g(-t) / E$, where $E=\int^{\infty} g^{2}(t) d t$, and sampled at the symbol rate to produce $M$ received sequences $r_{k}^{(1)}$ through $r_{k}^{(M)}$.

We make the flat-fading assumption that the frequency response $h_{i, j}(f)$ at receive antenna $i$ from transmit antenna $j$ does not differ appreciably from $h_{i, j}=h_{i, j}\left(f_{0}\right)$ over the signal band. We often make the independent Rayleigh-fading assumption that $\left\{h_{i, j}\right\}$ are i.i.d. zero-mean symmetric complex Gaussian random variables. We assume independent additive whiteGaussian noise at each receiver antenna with power spectral density $N_{0} / 2$. The average signal energy received at each receive antenna per signaling interval is $(E / 2) \mathrm{E}\left[\left\|\boldsymbol{x}_{k}\right\|^{2}\right] \mathrm{E}\left[\left|h_{i, j}\right|^{2}\right]$. Without loss of generality we normalize the symbols and channel so that $\mathrm{E}\left[\left\|\boldsymbol{x}_{k}\right\|^{2}\right]=\mathrm{E}\left[\left|h_{i, j}\right|^{2}\right]=1$, in which case the average SNR per bit per antenna reduces to $S N R_{b}=E /\left(2 R N_{0}\right)$,

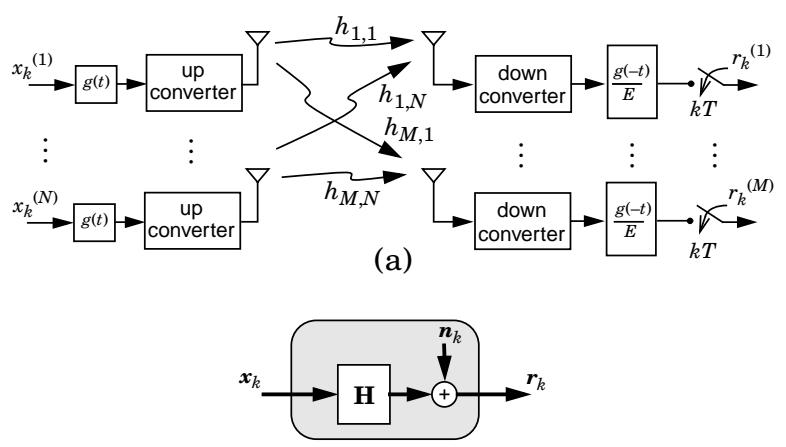

(b)

Fig. 1. An array-to-array communication link:

(a) passband model; (b) equivalent discrete-time model. 
where $R$ is the transmission rate in bits per signaling interval, or equivalently in bits $/ \mathrm{sec} / \mathrm{Hz}$.

The equivalent discrete-time baseband model is

$$
\boldsymbol{r}_{k}=\mathbf{H} \boldsymbol{x}_{k}+\boldsymbol{n}_{k},
$$

as shown in Fig. 1-b, where $\boldsymbol{x}_{k}=\left[x_{k}^{(1)}, \ldots, x_{k}^{(N)}\right]^{T}$ and $\boldsymbol{r}_{k}=\left[r_{k}{ }^{(1)}, \ldots, r_{k}{ }^{(M)}\right]^{T}$ are column vectors. Because of our normalization convention, the real and imaginary components of the noise vector $\boldsymbol{n}_{k}$ in (1) are i.i.d. zeromean Gaussian random variables with variance $\sigma^{2}=\left(2 R \cdot S N R_{b}\right)^{-1}$.

\section{Space-Time Block Codes}

The Alamouti space-time code achieves order- $2 M$ diversity with $N=2$ transmit antennas and with a simple detector. During the $2 k$-th and $2 k+1$-st signaling intervals, the signal vectors $\boldsymbol{x}_{2 k}=\left[a_{1}, a_{2}\right]^{T}$ and $\boldsymbol{x}_{2 k+1}=$ $\left[-a_{2}{ }^{*}, a_{1}{ }^{*}\right]^{T}$ are transmitted, where $\mathrm{E}\left[\left|a_{1}\right|^{2}\right]=\mathrm{E}\left[\left|a_{2}\right|^{2}\right]$ $=0.5$. The spectral efficiency is identical to that of an uncoded single-antenna transmitter using the same alphabet, since both deliver on average one symbol per signaling interval.

The two outputs of a matrix-matched filter are

$$
\begin{gathered}
z_{1}=\frac{1}{H_{F}} \sum_{i=1}^{M}\left(h_{i, 1}{ }^{*} r_{2 k}{ }^{(i)}+h_{i, 2}{ }^{*} r_{2 k+1}{ }^{(i)}\right)=H_{F} a_{1}+\eta_{1}, \\
z_{2}=\frac{1}{H_{F}} \sum_{i=1}^{M}\left(h_{i, 2} r_{2 k}{ }^{(i)^{*}}-h_{i, 1} r_{2 k+1}{ }^{(i)^{*}}\right)=H_{F} a_{2}+\eta_{2}
\end{gathered}
$$

where $H_{F}$ is the Frobenius norm of $\mathbf{H}$, and where the noise components $\eta_{1}$ and $\eta_{2}$ are i.i.d. with the same distribution as the noise components in (1), namely zero-mean complex Gaussian satisfying $\mathrm{E}\left[\left|\eta_{i}\right|^{2}\right]=$ $\left(R \cdot S N R_{b}\right)^{-1}$. The joint maximum-likelihood (ML) detector thus separates into a pair of scalar detectors, a result due to the orthogonality of the code.

If the number of transmit antennas is more than two, it has been shown that the spectral efficiency of
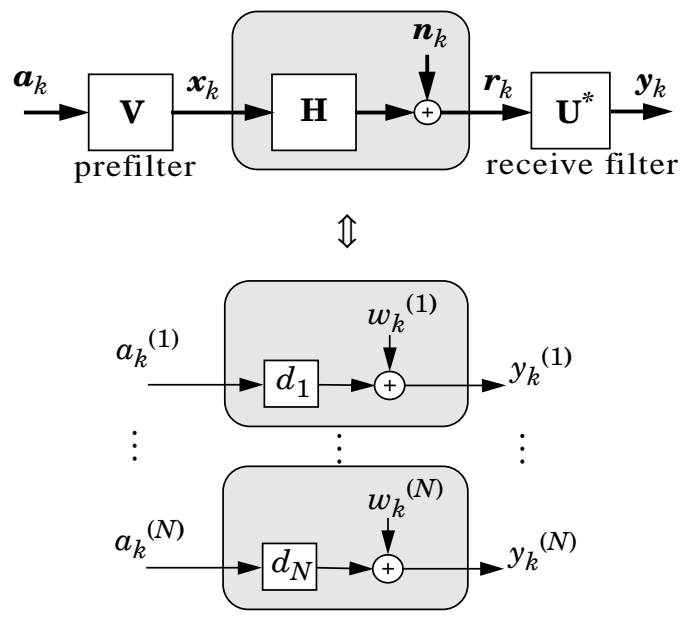

Fig. 2. The SVD Scheme. orthogonal STBC is strictly less than that of an uncoded single-antenna transmitter [5]. As an alternative, we consider the following nonorthogonal code for $N=4$ transmit antennas, which suffers no penalty in spectral efficiency:

$$
\left[\boldsymbol{x}_{4 k}, \boldsymbol{x}_{4 k+1}, \boldsymbol{x}_{4 k+2}, \boldsymbol{x}_{4 k+3}\right]=\left[\begin{array}{cccc}
a_{1} & -a_{2} & a_{3} & -a_{4} \\
a_{2} & a_{1} * & a_{4} & a_{3} \\
a_{3} & -a_{4}^{*} & a_{1} & -a_{2} \\
a_{4} & a_{3} * & a_{2} & a_{1}^{*}
\end{array}\right] .
$$

This code does incur a penalty in complexity, however. It can be shown that the joint ML detector reduces to a pair of ML detectors, with $a_{1}$ and $a_{3}$ detected independently of $a_{2}$ and $a_{4}$, where $\mathrm{E}\left[\left|a_{1}\right|^{2}\right]=\mathrm{E}\left[\left|a_{2}\right|^{2}\right]$ $=\mathrm{E}\left[\left|a_{3}\right|^{2}\right]=\mathrm{E}\left[\left|a_{4}\right|^{2}\right]=0.25$. Therefore, the detection complexity is proportional to the square of constellation size, unlike the linear complexity for orthogonal STBC.

\section{The SVD Scheme}

The space-time block codes described above require no knowledge of the channel at the transmitter. However, if the transmitter does have this knowledge, it can be exploited to approach Shannon capacity. Assume for simplicity that $\mathbf{H}$ is square $(M=N)$. Let $\mathbf{H}=\mathbf{U D V}^{*}$ be an SVD of $\mathbf{H}$, where $\mathbf{U}$ and $\mathbf{V}$ are unitary matrices and $\mathbf{D}$ is a diagonal matrix with non-decreasing nonnegative diagonal elements $\left\{d_{1}, d_{2}, \ldots, d_{N}\right\}$, the singular values. The asterisk denotes Hermitian transpose. When both the transmitter and the receiver know the channel, the channel matrix $\mathbf{H}$ can be decomposed into $N$ parallel subchannels by a prefilter $\mathbf{V}$ at the transmitter and a receive filter $\mathbf{U}^{*}$ at the receiver. The output of the receive filter is

$$
\boldsymbol{y}_{k}=\mathbf{U}^{*} \mathbf{H V} \boldsymbol{a}_{k}+\mathbf{U}^{*} \boldsymbol{n}_{k}=\mathbf{D} \boldsymbol{a}_{k}+\boldsymbol{w}_{k},
$$

where the noise $\boldsymbol{w}_{k}=\mathbf{U}^{*} \boldsymbol{n}_{k}$ is statistically identical to $\boldsymbol{n}_{k}$. This diagonalized system is illustrated in Fig. 2.

Although the singular values $\left\{d_{1}, d_{2}, \ldots, d_{N}\right\}$ are random variables, they are much more predictable than the $\left\{h_{i, j}\right\}$ of the underlying channel. In Fig. 3, we illustrate the Rayleigh pdf $p(t)=2 t e^{-t^{2}}$ for the random variable $\left|h_{i, j}\right|$ and also the estimated marginal pdf's for the five singular values of a $5 \times 5$ system. The second moment of the largest singular value is approximately $\mathrm{E}\left[d_{1}^{2}\right] \approx 13.1(11.2 \mathrm{~dB})$. In contrast, the second moment of $\left|h_{i, j}\right|$ is unity. The gain of $d_{1}$ relative to any $\left|h_{i, j}\right|$ can be interpreted as a form of diversity.

In order to compare the SVD scheme to Alamouti's scheme, a $2 \times 2$ system is considered. Recall that a symbol is transmitted twice in Alamouti's scheme, whereas it is transmitted once in the SVD scheme. It thus appears that the SVD offers a power advantage. Assume that we only use the first singular subchannel in the SVD scheme, which has a channel gain of $d_{1}$. It can 
be shown that $d_{1}$ is always bigger than $H_{F} / \sqrt{2}$, which is the channel gain of Alamouti's scheme (2)-(3). In fact, $d_{1}$ is closer to $H_{F}\left(\mathrm{E}\left[d_{1}{ }^{2}\right] \approx 3.5\right.$, whereas $\mathrm{E}\left[H_{F}{ }^{2}\right]=$ 4.0). Therefore, the power advantage of the SVD scheme over Alamouti's scheme approaches $3 \mathrm{~dB}$ in a 2 $\times 2$ system, even though the low-SNR subchannel (corresponding to the $d_{2}$ ) is not used.

By the invertible and norm-preserving properties of $\mathbf{U}$ and $\mathbf{V}$, the capacity of the system in Fig. 1-a is identical to that of the diagonalized system of Fig. 2. It is known that the capacity of the parallel subchannels is theoretically achieved by allocating power to each of the subchannels according to the water-pouring procedure. Many practical bit and power allocation algorithms based on the water-pouring procedure have been reported, some of which impose integer bit constraints.

For small $N$ and $R$, however, it is also feasible to allocate bits and power so as to minimize the vector symbol error probability (VSEP) $\operatorname{Pr}\left[\boldsymbol{a}_{k} \neq \hat{\boldsymbol{a}}_{k}\right]$ for the finite combinations of bit allocation. For example, $\{[4$ 0], [3 1], [2 2] \} is the set of possible bit allocations when $N=2$ and $R=4$. Increasing bit allocations (such as [1, 3]) are not considered because $d_{1} \geq d_{2}$. For a given bit allocation $\left\{b_{j}\right\}$, power is distributed according to the capacit $\mathrm{a}$ formula $p_{j}=2 \sigma^{2}\left(2^{b_{j}}-{ }^{\mathrm{a}} 1\right) / d_{j}^{2}$, and scaled to satisfy $\mathrm{E}\left[\left\|\boldsymbol{a}_{k}\right\|^{2}\right]=1$. Then, we choose the bit allocation that has the smallest VSEP. In some cases, the transmitter can avoid altogether dynamic allocation, by exploiting the reduced variability and decreasing nature of the singular values. Hence, bits and power may be distributed in a fixed manner regardless of subchannel gains. The performance degradation by using the fixed allocation is small, as illustrated in later results (see Fig. 4).

The most important advantage of the SVD scheme is that its performance approaches the theoretical capacity when combined with effective channel codes. Traditional one-dimensional channel codes are readily used instead of high-complexity space-time codes [3]. Another important advantage is the simple detection at the receiver, since proper prefilter and receive filter allow the detection of each subchannel to be independent of the others. The receiver complexity thus grows only linearly with the number of antennas and

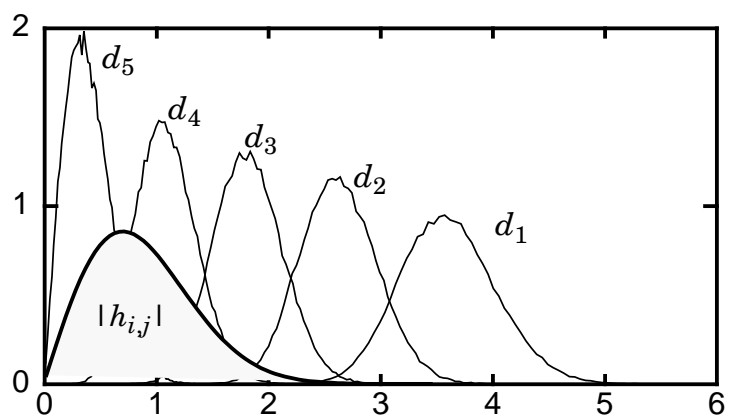

Fig. 3. Estimated pdf's for singular values. with the size of the alphabets. Moreover, it is easy to extend the SVD scheme for any $M$ and $N$, with $M$ not necessarily equal to $N$. In contrast, orthogonal STBC must sacrifice spectral efficiency for more than two transmit antennas.

\section{Numerical Results}

The discrete-time model in (1) was used to estimate an "SNR $R_{b}$ requirement" for the SVD scheme, which we define as the average $S N R_{b}$ required to achieve a BER of $10^{-4}$ using uncoded QAM alphabets ${ }^{1}$ and allocating bits so as to minimize VSEP. The results are summarized in Fig. 4 for a $2 \times 2$ system and a $4 \times 4$ system, considering spectral efficiencies in the range $R$ $\in\{2,3,4,5,6\}$. As a benchmark the SNR requirements for STBC are also shown, using Alamouti code for the $2 \times 2$ system, and using the nonorthogonal code in (4) for the $4 \times 4$ system.

The curves labeled "Fixed SVD" are the $S N R_{b}$ requirement for the SVD system with a fixed allocation of $\left\{\left[\begin{array}{ll}2 & 0\end{array}\right],\left[\begin{array}{ll}3 & 0\end{array}\right],\left[\begin{array}{ll}4 & 0\end{array}\right],\left[\begin{array}{ll}5 & 0\end{array}\right],\left[\begin{array}{ll}6 & 0\end{array}\right]\right\}$ for the $2 \times 2$ system, and $\left\{\left[\begin{array}{llll}2 & 0 & 0 & 0\end{array}\right],\left[\begin{array}{llll}3 & 0 & 0 & 0\end{array}\right],\left[\begin{array}{llll}2 & 2 & 0 & 0\end{array}\right],\left[\begin{array}{llll}3 & 2 & 0 & 0\end{array}\right],\left[\begin{array}{llll}4 & 2 & 0 & 0\end{array}\right]\right\}$ for the $4 \times 4$ system. These were the most frequently observed allocations under optimized allocation. For the $2 \times 2$ system, the fixed allocation incurs a penalty of between $0.1 \mathrm{~dB}$ and $1.3 \mathrm{~dB}$, while it is only between $0.1 \mathrm{~dB}$ and $0.4 \mathrm{~dB}$ for the $4 \times 4$ system.

It is seen that the SVD scheme significantly outperforms STBC. For example, at $S N R_{b}=14 \mathrm{~dB}$ for the $2 \times 2$ system, the Alamouti's scheme can convey only $R=4$ bits per signaling interval, whereas the SVD scheme can convey $R=6$. Similarly, at $S N R_{b}=3.4 \mathrm{~dB}$ for the $4 \times 4$ system, the STBC can convey $R=2$ bits per signaling interval, whereas the SVD scheme can convey over $R=6$ bits per signaling interval.

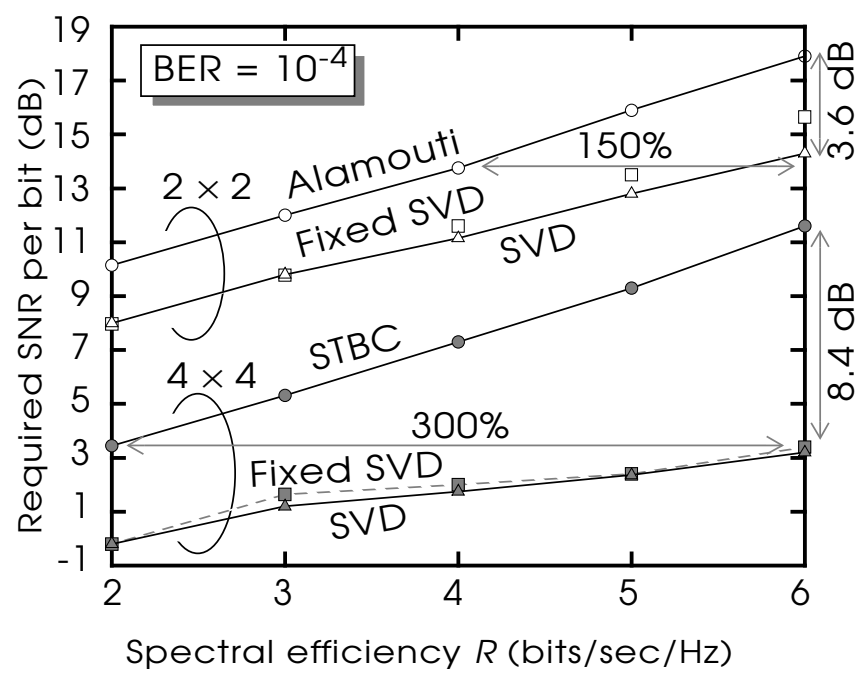

Fig. 4. Performance of the SVD scheme and STBC.

1. For $R=3$, hexagonal constellation was used for better performance. 
For the $2 \times 2$ system, the SNR gap between the fixed SVD scheme and Alamouti's scheme is around $2.5 \mathrm{~dB}$, a close match to $\mathrm{E}\left[d_{1}{ }^{2}\right] / \mathrm{E}\left[H_{F}{ }^{2} / 2\right] \approx 2.43 \mathrm{~dB}$. When dynamic allocation is used, this gap increases to more than $3.6 \mathrm{~dB}$ for $R=6$. The $S N R$ gap increases even more dramatically for the $4 \times 4$ system. If only the largest subchannel $\left(d_{1}\right)$ is used, the expected gain is $\mathrm{E}\left[d_{1}^{2}\right] / \mathrm{E}\left[H_{F}{ }^{2} / 4\right] \approx 3.9 \mathrm{~dB}$, which matches closely the $3.8 \mathrm{~dB}$ gain of the fixed SVD in Fig. 4 at $R=2$ and $R=4$. This SNR gap increases to $8.3 \mathrm{~dB}$ as $R$ increases from 2 to 6 , and as more and more subchannels are used.

For comparison, the orthogonal STBC for the $4 \times 4$ system with code rate of $1 / 2$ from [5] requires $S N R_{b}=$ $7.1 \mathrm{~dB}$ for $R=2(16 \mathrm{QAM})$ and $S N R_{b}=11.4 \mathrm{~dB}$ for $R$ $=3(64 \mathrm{QAM})$ to achieve a BER of $10^{4}$.

\section{Exploiting Reciprocity with TDD}

If the receiver estimates the channel matrix $\mathbf{H}$ and performs an SVD of $\mathbf{H}$, then the prefilter information may be sent to the transmitter via a feedback link. In a TDD system, however, the use of feedback may be avoided by exploiting the reciprocity of wireless channels. In particular, the receive filter may be estimated while receiving signals from the other end, and the estimated receive filter may be used as the prefilter during transmission in the opposite direction. As shown in Fig. 5, let the link from left to right be the forward link, and let the link from right to left be the reverse link. Further, let us use column vectors and row vectors, respectively, to represent signals in the forward and reverse paths. With this convention, reciprocity implies that the forward and reverse paths both see the same channel matrix $\mathbf{H}$. Suppose that $\mathbf{V}$ and $\mathbf{U}^{*}$ are the optimal prefilter and receive filter, respectively, in the forward link as represented in (5). It then follows that, in the reverse link, $\mathbf{U}^{*}$ is the optimal prefilter and $\mathbf{V}$ is the optimal receive filter. Specifically, the reverse link is diagonalized according to:

$$
\tilde{\boldsymbol{y}}_{k}^{T}=\widetilde{\boldsymbol{a}}_{k}^{T} \mathbf{U}^{*} \mathbf{H V}+\widetilde{\boldsymbol{n}}_{k}^{T} \mathbf{U}^{*}=\widetilde{\boldsymbol{a}}_{k}^{T} \mathbf{D}+\widetilde{\boldsymbol{n}}_{k}^{T} \mathbf{U}^{*} .
$$

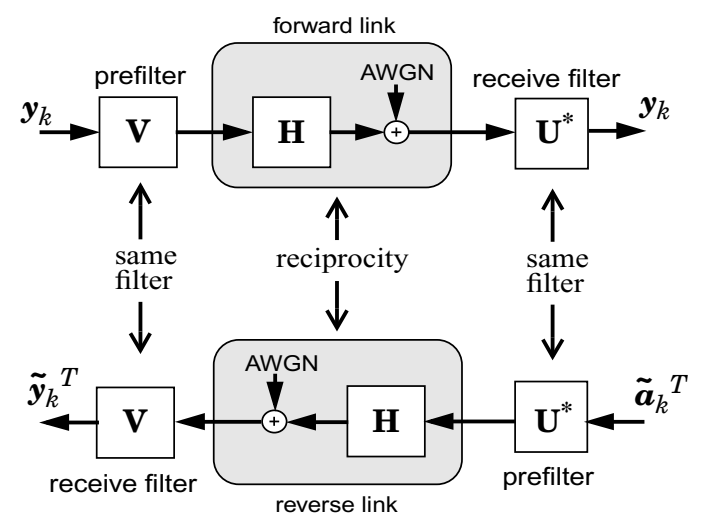

Fig. 5. The SVD scheme in a TDD system.
Hence, if the receive filters are estimated during reception, they may be used as prefilters without change during transmission.

An intuitive method to estimate the receive filter is to compute the SVD of an estimate of $\mathbf{H}$ directly. Other methods are based on an eigendecomposition of the covariance matrix of $\boldsymbol{r}_{k}$ :

$$
\mathbf{R}_{r}=\mathbf{E}\left[\boldsymbol{r}_{k} \boldsymbol{r}_{k}{ }^{*}\right]=\mathbf{U D}^{2} \mathbf{U}^{*}+2 \sigma^{2} \mathbf{I}_{M},
$$

where $\mathbf{I}_{M}$ is an $M \times M$ identity matrix, which requires that the transmitter distribute power equally to all antennas. Algorithms based on eigendecomposition can be implemented adaptively and blindly, so that explicit channel estimation is not necessary.

\section{Conclusions}

When the transmitter knows the channel, the optimal space-time processing strategy is based on an SVD. We have shown by simulations that the SVD scheme without channel coding can significantly outperform STBC, achieving $150 \%$ and $300 \%$ higher transmission rates for $2 \times 2$ systems and $4 \times 4$ systems, respectively. We observed a small penalty by using a fixed bit and power allocation. Implementation of the SVD scheme was seen to simplify in TDD systems. The concepts presented in this paper may be extended to frequency-selective channels by combining them with orthogonal frequency-division multiplexing.

\section{References}

[1] G. J. Foschini, Jr., and M. J. Gans, "On limits of wireless communication in a fading environment when using multiple antennas," Wireless Personal Commun., vol. 6, no. 3, pp. 311335, Mar. 1998.

[2] G. J. Foschini, Jr., "Layered space-time architecture for wireless communication in a fading environment when using multi-element antennas," Bell Labs Tech. Journal, vol. 1, no. 2, pp. 41-59, Autumn 1996.

[3] V. Tarokh, N. Seshadri, and A. R. Calderbank, "Space-time codes for high data rate wireless communication: performance criteria and code construction," IEEE Trans. Information Theory, vol. 44, no. 2, pp. 744-765, Mar. 1999.

[4] S. M. Alamouti, "A simple transmitter diversity scheme for wireless communications," IEEE J. Select. Areas Commun., vol. 16, no. 8, pp. 1451-1458, Oct. 1998.

[5] V. Tarokh, H. Jafarkhani, and A. R. Calderbank, "Space-time block codes from orthogonal designs," IEEE Trans. Information Theory, vol. 45, no. 5, pp. 1456-1467, Jul. 1999.

[6] V. Tarokh, H. Jafarkhani, and A. R. Calderbank, "Space-time block codes for wireless communications: performance results," IEEE J. Select. Areas Commun., vol. 17, no. 3, pp. 451-460, Mar. 1999.

[7] L. H. Brandenburg, and A. D. Wyner, "Capacity of the Gaussian channel with memory: the multivariate case," Bell Syst. Tech. J., vol. 53, no. 5, pp. 745-778, May/Jun. 1974.

[8] A. Reial, and S. G. Wilson, "Capacity-maximizing transmitter processing for fading matrix channel," Commun. Theory Mini Conf. 1999, pp. 6-10, Jun. 1999. 